\title{
A General Design and Optimization Method of Tightly-Coupled Cross-Dipoles for Base Station
}

\author{
Can Ding ${ }^{1}$, Haihan Sun $^{1}$, Y. Jay Guo ${ }^{1}$, Richard W. Ziolkowski ${ }^{1,2}$ \\ ${ }^{1}$ Global Big Data Technologies Centre (GBDTC), University of Technology Sydney (UTS), \\ Sydney, NSW 2007, Australia, can.ding@uts.edu.au \\ ${ }^{2}$ Department of Electrical and Computer Engineering, The University of Arizona, \\ Tucson, AZ 85721, USA, ziolkows@email.arizona.edu.
}

\begin{abstract}
This paper investigates the working mechanism of dual-polarized tightly-coupled cross-dipoles that are widely used in cellular base station applications. The effects of couplings between sub-dipoles on the performance indexes of concern are observed. A theory of considering this type of cross-dipole as an array is proposed and validated. The proposed theory explains why a stable radiation pattern can be achieved by this kind of structure. The array model can be used to guide the introduction and optimization of a simplified cross-dipole structure for base station application.
\end{abstract}

Index Terms-Cross-dipole, base station, array, half-powerbeamwidth (HPBW).

\section{INTRODUCTION}

Our daily lives have benefited significantly from the development of wireless communication technologies. Modern communication systems provide us with not only communication, but also other applications such as internet connectivity, video conferences, and virtual reality. The upcoming 5th generation $(5 \mathrm{G})$ wireless communication system will enable more revolutionary technologies like home service robotic, autonomous car to be available in daily life. To build $5 \mathrm{G}$ wireless communication systems, one of the major challenges is to deploy future $5 \mathrm{G}$ antennas and $3 \mathrm{G} / 4 \mathrm{G}$ antennas together on a new platform. This will leads to more stringent requirements on base station antennas due to the complicated environment. It is now becoming imperative for the mobile industry to develop new antenna technologies to address the challenges in deploying $5 \mathrm{G}$ base stations antennas. Due to the fact that $5 \mathrm{G}$ standardization on base station antennas has not finished yet, the most effective starting point is the investigation of current base station antennas on $3 \mathrm{G} / 4 \mathrm{G}$ platforms. Beholding a knowledge of how current base station antenna works can provide significant help to re-design base station antennas on the new platform.

Current base station antennas for wireless cellular communication are designed to provide dual $\pm 45^{\circ}$ polarizations to enhance the system capacity. To provide a full coverage of a geographic area, usually 3 arrays are employed to have an omnidirectional pattern in the horizontal plane and a narrow beam in the vertical plane. The base station elements used in this typical 3-sector configuration are required to have stable radiation pattern in the horizontal plane across a wide operation band, to be specific, HPBW within $65^{\circ} \pm 5^{\circ}$ over

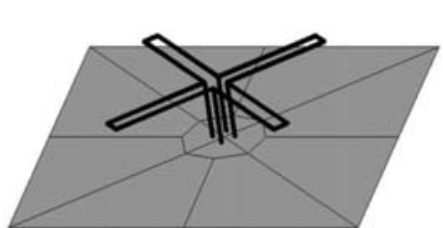

(a)

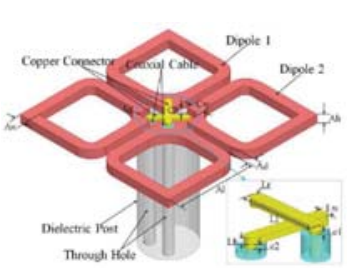

(c)

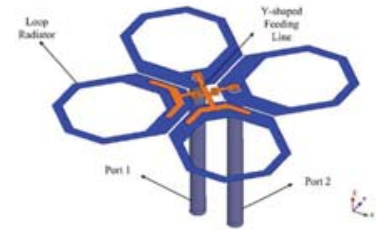

(b)

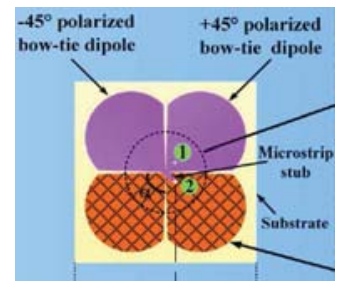

(d)
Fig. 1. Configurations of dual-polarized cross-dipoles developed for base station applications reported in the literature. (a) Ref [4]. (b) Ref [5]. (c) Ref [6]. (d) Ref [7].

the band from $1710 \mathrm{MHz}$ to $2690 \mathrm{MHz}$. Moreover, excellent matching of VSWR $<1.5$ for both of the two polarizations is strictly required in industry.

There are various types of antennas targeted at this application in the literature, including patch antennas [1], MagnetoElectric dipoles [2], slot antennas [3], and cross-dipoles [4-7]. However, only a few works can meet all the requirements that have the potential to be used commercially. Among those works, cross-dipoles appear to be the most promising solutions due to their wide bandwidth and stable radiation pattern. Fig. 2 illustrates some cross-dipoles available in the literature [4-7]. A dual-polarized cross-dipole consists of a pair of sub-dipoles perpendicular to each other. By exciting either one of the two sub-dipoles, different polarizations can be obtained. Primitive cross-dipoles have their two sub-dipoles "isolated" from each other, like the designs shown in Fig. 1(a). These "isolated" configurations have limited bandwidth. Lately, cross-dipoles with their sub-dipoles closely spaced and tightly coupled with each other are advocated, as shown in Fig. 1(b) to 1(d). By optimally enhancing the mutual coupling between subdipoles, both the two sub-dipoles can be activated when only one sub-dipole is excited. This somehow leads to a fact that 


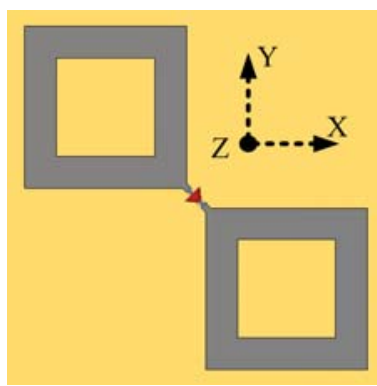

(a)

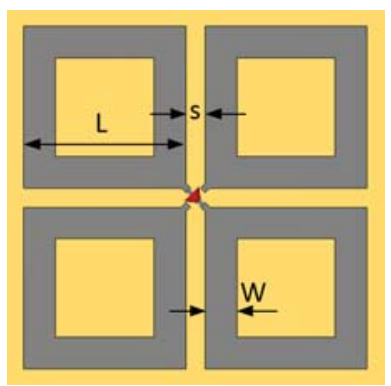

(b)
Fig. 2. Cross-dipole geometry. (a) Model-A: tightly-coupled cross dipoles consisting of a driven dipole and a parasitic dipole. (b) Model-B: driven dipole only.

the impedance bandwidths have been significantly improved. For example, all the reported antennas shown in Fig. 1(b) to 1(d) [5-7] have their bandwidths $>44.5 \%$ with VSWR $<1.5$. Moreover, we noticed that a stronger coupling between subdipoles may also increase the gain.

Now it is noted that the coupling between sub-dipoles gives a cross-dipole more bandwidth and better radiation performance. However, the understanding of how the coupling improves the performance is superficial. There is no clear methodology available of how to optimize the coupling to achieve the best performance. This work offers a deeper understanding of how coupling works and provides linkages between physical dimensions, current distribution, and performance indexes of concern. Substantial simulation and analysis have been conducted to support the proposed theory. Based on the proposed theory, a design and optimization method of a simplified tightly-coupled cross-dipole is obtained.

\section{Coupling Analysis}

The analysis in this work is based on a cross-dipole with a typical configuration as shown in Fig. 2(a). It consists of two identical sub-dipoles placed perpendicular to each other. Square-looped dipole arms are employed in order to have a larger aperture to get more bandwidth and gain. The branches constructing the square-looped arms have the length of $L=$ $25.5 \mathrm{~mm}$ and the width of $W=6 \mathrm{~mm}$. The square-looped arms are placed close to each other with a small distance of $s=3 \mathrm{~mm}$ to enable strong coupling. The dipoles are printed on a Rogers substrate with the dielectric constant of 3.55 and the thickness of $1.524 \mathrm{~mm}$. The distance between the dipole and the reflector is $h=32 \mathrm{~mm}$ and the reflector has the size of $G=160 \mathrm{~mm}$.

The data used for theoretical analysis are obtained without the presence of balun and matching circuit. This is due to the fact that the matching circuit will be shielded by the balun in reality and a well-designed balun has minor current on its outer surface, thereby they have minor effect on the radiation performance. The balun and matching circuit are only used for impedance matching and balance feeding.

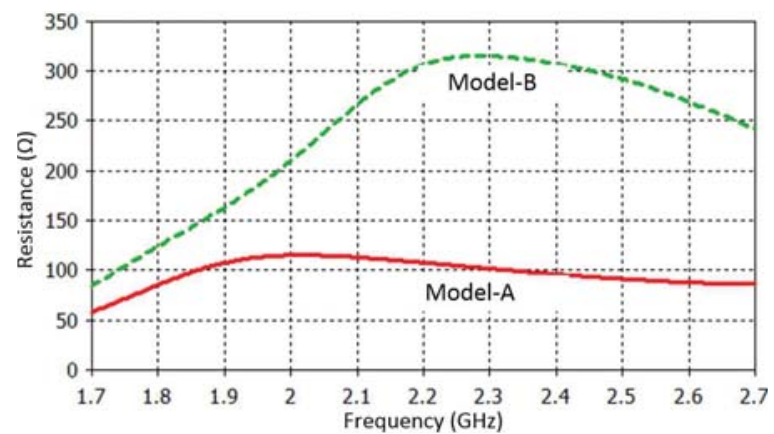

(a)

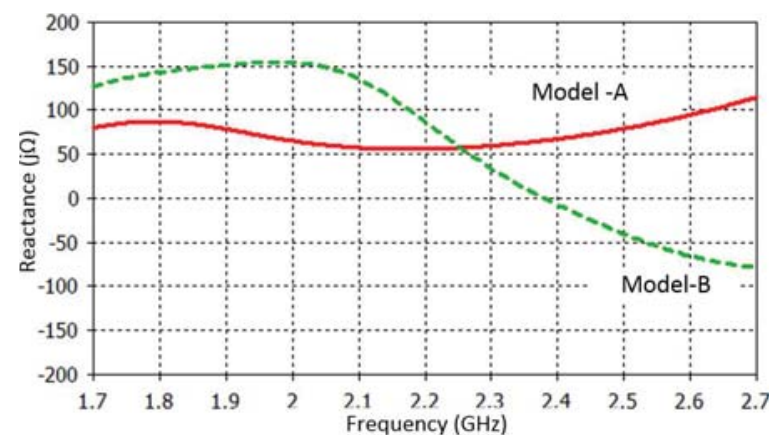

(b)

Fig. 3. Variations of the impedance of Model-A and Model-B in the target band. (a) Resistance. (b) Reactance.

The two sub-dipoles shown in Fig. 2(a) are defined as driven dipole and parasitic dipole. By exciting the driven dipole, the parasitic dipole can also be activated due to the coupling. To figure out how the coupling changes the performance, we built another model by removing the parasitic dipole [as shown in Fig. 2(b)] and investigated the differences between these two models.

Fig. 3(a) and 3(b) shows the variations of resistance and reactance, respectively, of Models- $\mathrm{A}$ and $-\mathrm{B}$ across the designed band. It is observed that the fluctuations in both the resistance and the reactance are smaller with the presence of the parasitic dipole. This indicates the fact that the parasitic dipole alleviates the impedance variation of the driven dipole, making the dipole easier to match in the designed band. This is under our expectation since the dipole's size is enlarged due to presence of the parasitic dipole, which introduces more bandwidth.

Moreover, the coupling between driven and parasitic dipoles offers a higher directivity and a more stable HPBW in the horizontal plane cut ( $x z$-plane). From $1.7 \mathrm{GHz}$ to $2.7 \mathrm{GHz}$, the model shown in Fig. 2(a) has its directivity varying from 8.6 $\mathrm{dBi}$ to $9.3 \mathrm{dBi}$, and the $\mathrm{HPBW}$ is from $63^{\circ}$ to $69^{\circ}$, while those of the model shown in Fig. 2(b) are from $8.5 \mathrm{dBi}$ to $9.0 \mathrm{dBi}$ and from $65^{\circ}$ to $75^{\circ}$, respectively. To have a better understanding of where these merits come from, the current distribution on the apertures is depicted in Fig. 4(a) and 4(b). It is observed that the driven dipole has the standard dipole behavior with or without the coupling. On the driven dipole, the peaks of 


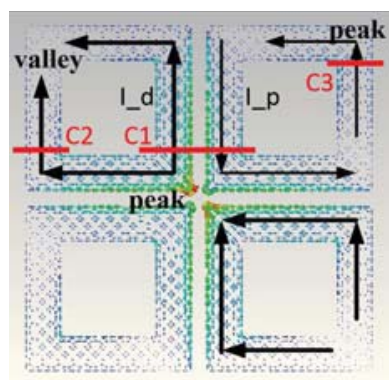

(a)

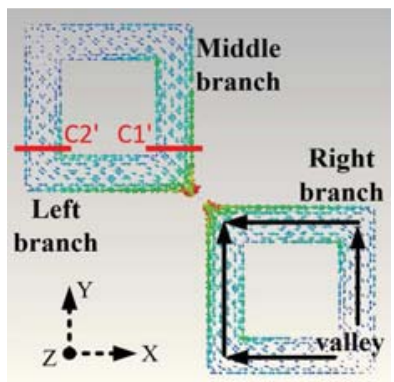

(b)
Fig. 4. Current distributions on the traces of the models. (a) Model-A. (b) Model-B.

the current distribution occur near the excitation source and the valleys locate at the ends of the dipole arms. The parasitic dipole has a different current distribution since it is illuminated by the capacitive coupling between the parallel branches. We monitored the currents on a pair of adjacent branches as a typical example, finding out that the induced current $I \_$and the driven current $I \_d$ are out of phase. This phenomenon is interesting since in theory, the reverse current can reduce the directivity while in reality, the directivity is even higher. In order to explain this conflict, several current monitors $(\mathrm{C} 1$, $\mathrm{C} 2, \mathrm{C} 3, \mathrm{C} 1$ ' and $\mathrm{C} 2$ ') were applied to the dipoles (as shown in Fig. 4) to observe both the magnitudes and phases of the currents within the entire frequency band. Due to the diagonal symmetric aperture, the current distribution on the $\mathrm{x}$ - and $\mathrm{y}-$ aligned branches is identical. Therefore, only the y-aligned currents are monitored and analyzed. The y-aligned currents on the left-, middle-, and right branches work together like a 3-elements array. The current monitors are placed near the peaks of the current distribution on the three branches.

The current magnitudes on the middle branches of the two models at $\mathrm{C} 1$ and $\mathrm{C} 1$ ' are compared in Fig. 5(a). The comparison of the current magnitudes on the left branches at $\mathrm{C} 2, \mathrm{C} 2$ ' and that on the right branch at C3 is plotted in Fig. 5(b). Following facts are noticed by observing the figures. Firstly, the magnitudes of the total currents $\left(I \_d-I p\right)$ on the middle branch with or without the coupling are at the same level $(\mathrm{C} 1 \approx \mathrm{C} 1$ '). Although the coupling introduces a reverse current $I p$ on the parasitic dipole, the current density on the driven dipole $I \_d$ is also increased, thereby avoiding a reduction in the directivity. Secondly, the current on the left branch of the driven dipole remains at a similar level (C2 $\approx \mathrm{C} 2$ ') as well with or without the coupling. Thirdly, additional current (C3) is induced on the right branch. And the induced current has a comparable magnitude with the driven current on the left branch ( $\mathrm{C} 3$ comparable with $\mathrm{C} 2$ ). Moreover, the phases of the currents on the left-, middle, and right-branches of Model-A are plotted in Fig. 5(c). The phase differences between the currents are less than $90^{\circ}$ within the entire band. Therefore, the three y-aligned currents' contribution to the radiation adds up at the boreside. This leads to the fact that the coupling of Model-A introduces an additional current item

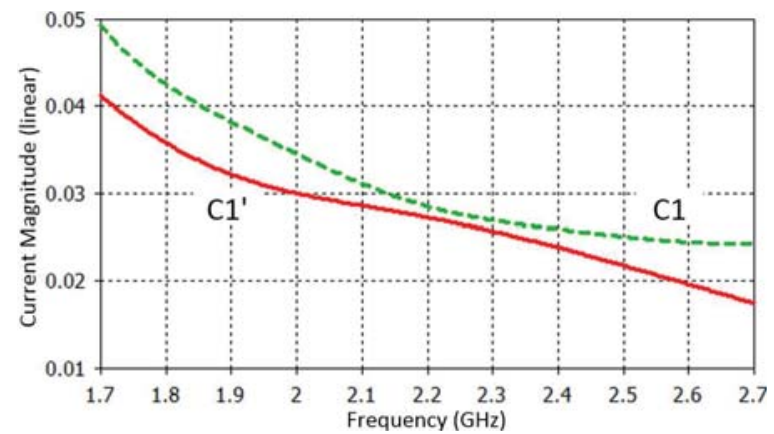

(a)

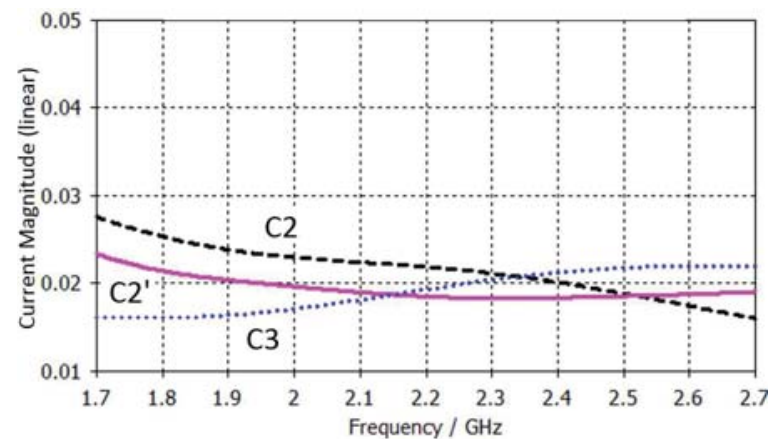

(b)

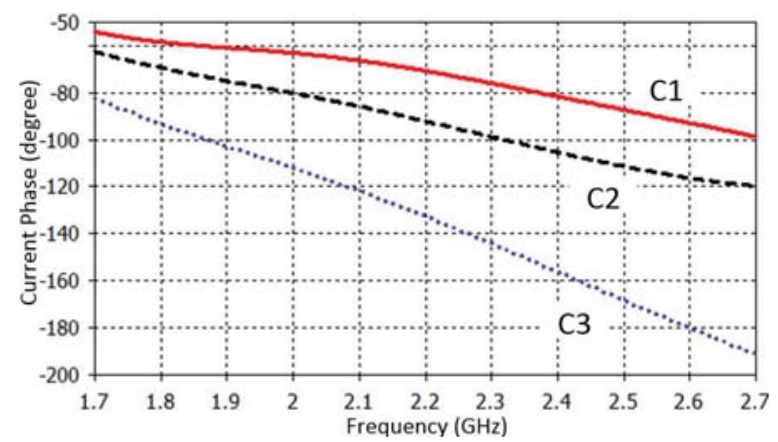

(c)

Fig. 5. Simulated currents. (a) Current magnitude on the middle branches of Model-A and Model-B. (b) Current magnitudes on the side branches of Model-A and Model-B. (c) Current phases on three different branches of Model-A.

(C3) without decreasing the driven current items (C1 and C2), resulting in a higher directivity and a narrower HPBW.

\section{EQuivalent Model}

Based on the analysis in the previous section, this section shows how to play with the aperture of a cross-dipole configuration to achieve stable HPBW across the wide band. Fig. 6(a) gives a schematic current distribution on the cross-dipole aperture. The solid and dashed arrows denote the directly-excited currents and the induced parasitic currents, respectively. Due to the symmetry, there are only three different current items 


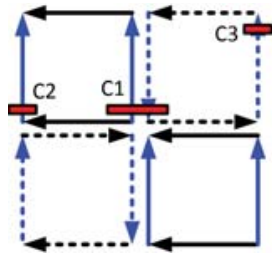

(a)

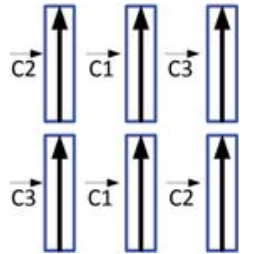

(b)

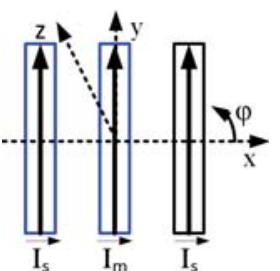

(c)
Fig. 6. Representation of the current distributions. (a) Schematic for the tightly-coupled cross-dipole antenna. (b) Equivalent six-element dipole array for model-A. (c) Reduced, equivalent three-element dipole array model.

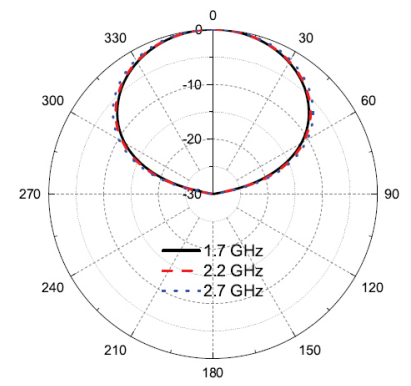

(a)

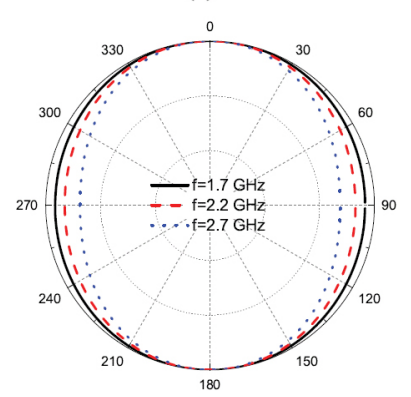

(c)

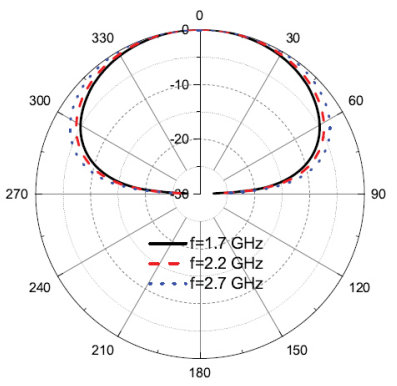

(b)

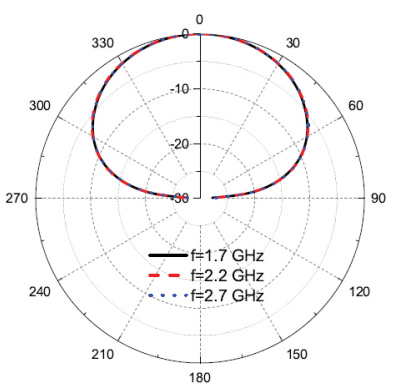

(d)
Fig. 7. Simulated radiation patterns. (a) E-plane pattern and (b) H-plane pattern of a single half-wavelength dipole placed above an infinite ground plane. (c) Three-element array factor pattern alone. (d) Model-A-based crossdipole radiation pattern.

on the aperture:

$$
\begin{aligned}
& \overrightarrow{C 1}=C 1 e^{j \phi_{1}}, \\
& \overrightarrow{C 2}=C 2 e^{j \phi_{2}}, \\
& \overrightarrow{C 3}=C 3 e^{j \phi_{3}} .
\end{aligned}
$$

Three current monitors $\mathrm{C} 1, \mathrm{C} 2$, and $\mathrm{C} 3$ are employed as shown in Fig. 6(a) to monitor the magnitudes and phases at the peaks of the currents. In this work, we take out only the yaligned currents to conduct the analysis as shown in Fig. 6(b). Although the three current items have different magnitudes and phases, they have similar quarter-period quasi-sinusoidal magnitude distribution and small phase differences $<90^{\circ}$ as long as the size of the cross-dipole is reasonable (side length of the aperture near a quarter-wavelength). Therefore, we can merge the six current items into three current items as shown in Fig. 6(c) and assume that the current items $\overrightarrow{I_{m}}$ and $\overrightarrow{I_{s}}$ have identical half-period quasi-sinusoidal current distribution, where

$$
\begin{array}{r}
\overrightarrow{I_{s}}=\overrightarrow{I_{0}}\left(C 3 e^{j \phi_{3}}+C 2 e^{j \phi_{2}}\right) / 2, \\
\overrightarrow{I_{m}}=\overrightarrow{I_{0}} C 1 e^{j \phi_{1},}
\end{array}
$$

The radiation pattern generated by the three y-polarized current items can be derived as

$$
F_{y}(\theta, \phi)=A F(\theta, \phi) * f_{0}(\theta, \phi),
$$

where $\operatorname{AF}(\theta, \phi)$ is the three-element array factor and $f_{0}(\theta, \phi)$ is the radiation pattern of a dipole element. Since the $\mathrm{x}$ - and $y$-aligned currents are rotationally symmetric, then we can have the radiation pattern contributed by $\mathrm{x}$-algined currents as follow:

$$
F_{x}(\theta, \phi)=F_{y}\left(\theta, \phi+90^{\circ}\right) .
$$

Therefore, combining the fields radiated by both the $x$ - and $y$-aligned currents, the total radiation pattern is:

$$
\begin{aligned}
F(\theta, \phi) & =F_{x}(\theta, \phi)+F_{y}(\theta, \phi) \\
& =F_{y}\left(\theta, \phi+90^{\circ}\right)+F_{y}(\theta, \phi) .
\end{aligned}
$$

The $x z$-cut of this radiation pattern is

$$
\begin{aligned}
F(\theta, \phi=0) & =F_{y}\left(\theta, \phi=0^{\circ}\right)+F_{y}\left(\theta, \phi=90^{\circ}\right) \\
& =A F\left(\theta, \phi=0^{\circ}\right) * f_{0}\left(\theta, \phi=0^{\circ}\right) \\
& +A F\left(\theta, \phi=90^{\circ}\right) * f_{0}\left(\theta, \phi=90^{\circ}\right) \\
& =A F\left(\theta, \phi=0^{\circ}\right) * f_{H}(\theta)+A F\left(\theta, \phi=90^{\circ}\right) * f_{E}(\theta)
\end{aligned}
$$

where $f_{H}(\theta)$ and $f_{E}(\theta)$ represent the H- and E-plane patterns of the dipole oriented along $\overrightarrow{I_{0}}$. Because the $y$-aligned currents are spaced along the $x$-axis, the array factor along the $y$-axis is identically equal to 1.0 , i.e.,

$$
A F\left(\theta, \phi=90^{\circ}\right) \equiv 1 .
$$

Therefore, the cross-dipole's radiation pattern in the $x z$-cut (6) can be rewritten as

$$
\left.F(\theta, \phi)\right|_{\phi=0^{\circ}}=A F\left(\theta, \phi=0^{\circ}\right) * f_{H}(\theta)+f_{E}(\theta) .
$$

where $A F_{H}$ is the three-dipole element array factor in the dipole's H-plane. The first and second terms in this expression represent the contributions from the $x$-and $y$-aligned dipole currents, respectively.

From equation (2) and Fig. 6(c), the three-element dipole array factor is calculated as:

$$
\begin{aligned}
A F(\theta) & =\left|\sum_{n=1}^{3} W_{n} e^{-j k \cdot r_{n}}\right| \\
& =\left|I_{0} e^{j \phi_{m}}\left[A_{s} e^{j\left(\phi_{s}-\phi_{m}\right)}\left(e^{-j k d \cos \theta}+e^{j k d \cos \theta}\right)+A_{m}\right]\right| \\
& =I_{0}\left|2 A_{s} \cos (k d \cos \theta) e^{j(\Delta \phi)}+A_{m}\right| \\
& =I_{0}\left\{\left[2 A_{s} \cos (k d \cos \theta) \cos (\Delta \phi)+A_{m}\right]^{2}\right. \\
& \left.+\left[2 A_{s} \cos (k d \cos \theta) \sin (\Delta \phi)\right]^{2}\right\}^{\frac{1}{2}}
\end{aligned}
$$

where $k=2 \pi / \lambda, \Delta \phi=\phi_{s}-\phi_{m}$, and $d$ is the separation distance between the dipole elements. The array factor is thus 
calculated straightforwardly once the magnitudes and phases of the currents are obtained from the monitors $\mathrm{C} 1, \mathrm{C} 2$, and C3.

Fig. 7(a) and 7(b) shows the E- and H-plane radiation patterns $\left(f_{E}(\theta)\right.$ and $\left.f_{H}(\theta)\right)$, respectively, of a straight halfwavelength dipole placed above a ground plane. To eliminate the effect of the ground plane's size, the ground plane used for this demonstration is set to be infinitely large. Fig. 7(c) plots the array factors of Model-A at different frequencies. The obtained array factors were calculated from the values of the current monitors and formula (17). It is observed from these figures that a typical dipole placed above a reflector has its H-plane pattern getting wider and E-plane pattern relatively stable with the increasing frequency. It is also noticed that array factor $A F_{H}(\theta)$ is narrower at a higher frequency. Therefore, according to equation (8), it is not surprising that the proposed cross-dipole has very stable radiation patterns across the target band.

\section{Discussion And Design Procedure}

The tightly-coupled cross-dipole configuration addressed in this work can be decomposed into two arrays, a $x$ - and a $y$-aligned 3-elements array, placed above a reflector. The radiation pattern is determined by two factors: a) the imaging factor determining each of the array element's radiation pattern and b) the array factor $A F_{H}(\theta)$. This array factor and imaging factor have opposite effects to the radiation pattern of the antenna. With the frequency increasing, the imaging effect makes the pattern wider, while the array effect narrowing the pattern. By manipulating the weighting of this two factors, beam consistency can be achieved.

For a typical cross-dipole model shown in Fig. 2, the basic parameters we can play with are the aperture length $L$, branch width $W$, gap width $s$, antenna height $h$, and reflector size $G$. According to our simulations, among those parameters, the most sensitive one is the dipole length $L$. This is attributed to the fact that $L$ is closely related to the array factor $A F_{H}(\theta)$ since $L / 2$ is the separation distance between array elements. A larger $L$ results in a more directive $A F_{H}(\theta)$, and thus a narrower HPBW. At the same time, $L$ is also a key parameter to tune the impedance.

The radiation pattern is also changing with antenna height $h$ and the ground plane size $G$. This is due to the fact that $h$ and $G$ are crucial to the radiation pattern $\left(f_{E}(\theta)\right.$ and $\left.f_{H}(\theta)\right)$ of a single dipole element; in other words, imaging factor. However, they do not affect the couplings happened on the aperture. This leads to the following two facts: first, they do not affect the array factor $A F_{H}(\theta)$; second, changing these two parameters in reasonable ranges do not affect impedance.

For parameters $s$ and $W$, they demonstrate minor capability of tuning the radiation pattern. This fact is easy to understand since the radiation pattern of a dipole is much related to the dipole length rather than the dipole width. However, optimizing the width is crucial for impedance matching.

Based on the obtained analysis results, a following design procedure is advocated. Firstly, $L, h$, and $G$ were determined to have a satisfactory radiation performance. The antenna height $h$ and reflector size $G$ were kept as small as possible to guarantee a compact structure. Parasitic elements maybe employed to better shape the beam. Secondly, $s$ and $W$ were optimized to have a matchable input impedance. To determine whether the realized cross-dipoles can be matched in the target band, a S1p file representing the input impedance was extracted from CST Microwave Studio. Then the S1p file can be connected with ideal matching circuit based on filter design. This matching method described in [8] is an optimal way to match dipole antennas and is easy to be implemented on PCB. Future work will show how a cross-dipole with excellent performance are obtained following this design procedure.

\section{CONCLUSION}

This paper is focused on analyzing the dual-polarized tightly-coupled cross-dipole which is widely used in current $2 \mathrm{G} / 3 \mathrm{G} / 4 \mathrm{G}$ base stations. An insight of how this type of antenna works is given by decomposing the cross-dipole into two arrays. By observing and studying the current distribution on the aperture, it is found that the radiation performance of such an antenna is determined by two factors, the imaging factor and the array factor. The interaction between these two factors makes the cross-dipole be able to provide very stable radiation performance, which is required for base station application. Then a strategy of tuning the weighting of these two factors is proposed followed by advocated design procedure. The knowledge generated in this work is not only a guidance of designing current base station antenna, but also useful for future $5 \mathrm{G}$ base station antennas.

\section{REFERENCES}

[1] Y. Jin and Z. Du, "Broadband Dual-Polarized F-Probe Fed Stacked Patch Antenna for Base Stations," IEEE Antennas and Wireless Propagation Letters, vol. 14, pp. 1121-1124, 2015.

[2] K. M. Luk and B. Wu, "The Magnetoelectric Dipole - A Wideband Antenna for Base Stations in Mobile Communications," Proceedings of the IEEE, vol. 100, no. 7, pp. 2297-2307, July 2012.

[3] R. Lian, Z. Wang, Y. Yin, J. Wu and X. Song, "Design of a Low-Profile Dual-Polarized Stepped Slot Antenna Array for Base Station," IEEE Antennas and Wireless Propagation Letters, vol. 15, pp. 362-365, 2016.

[4] H. D. Hristov, H. Carrasco, R. Feick, and B. L. Ooi, "Low-profile $\mathrm{X}$ antenna with flat reflector for polarization diversity applications," Microwave and Optical Technology Letters, vol. 51, pp. 1508-1512, 2009.

[5] Q. X. Chu, D. L. Wen and Y. Luo, "A Broadband $\pm 45^{\circ}$ Dual-Polarized Antenna With Y-Shaped Feeding Lines," IEEE Transactions on Antennas and Propagation, vol. 63, no. 2, pp. 483-490, Feb. 2015.

[6] Z. Bao, Z. Nie and X. Zong, "A Novel Broadband Dual-Polarization Antenna Utilizing Strong Mutual Coupling," IEEE Transactions on Antennas and Propagation, vol. 62, no. 1, pp. 450-454, Jan. 2014.

[7] Y. Cui, R. Li and H. Fu, "A Broadband Dual-Polarized Planar Antenna for 2G/3G/LTE Base Stations," IEEE Transactions on Antennas and Propagation, vol. 62, no. 9, pp. 4836-4840, Sep. 2014.

[8] C. Ding, B. B. Jones, Y. J. Guo, and P.-Y. Qin, "Wideband Matching of Full-Wavelength Dipole with Reflector for Base Station, "IEEE Transactions on Antennas and Propagation, vol. 65, no. 10, pp. 55715576, Oct. 2017. 